\title{
Maternal depression and child development: Evidence from São Paulo's Western Region Cohort Study
}

\author{
Alexandra Brentani ${ }^{1 *}$, Günther FinK² \\ ${ }^{1} \mathrm{PhD}$, Department of Pediatrics, Faculdade de Medicina, Universidade de São Paulo, São Paulo, SP, Brazil \\ ${ }^{2} \mathrm{PhD}$, Department of International Health Economics, Harvard School of Public Health, Cambridge, MA, USA
}

Study conducted at Department of Pediatrics, Faculdade de Medicina, Universidade de São Paulo (FMUSP), São Paulo, SP, Brazil

Article received: 6/29/2016 Accepted for publication: $7 / 26 / 2016$

*Correspondence: Departamento de Pediatria Address: Av. Dr. Arnaldo, 455

São Paulo, SP - Brazil

Postal code: 01246-903

brentani.alexandra@gmail.com

http://dx.doi.org/10.1590/1806-9282.62.06.524

Financial support: The project was funded by Secretaria Estadual da Saúde do Estado de São Paulo (SESSP)

\section{INTRODUCTION}

Healthy child development has been becoming increasingly postulated as instrumental for reducing economic and health inequities over the life course. ${ }^{1}$ As highlighted by the recent Lancet series, mental health has become one of the greatest challenges for global well-being, ${ }^{2}$ with depression as the most common and debilitating condi-
Introduction: While a growing body of evidence has investigated the relationship between maternal mental health and child development, evidence on children's early life outcomes remains mixed. We analyze the empirical relationship between maternal depression and children's development at age one using data from the São Paulo Western Region Cohort project.

Method: Seven hundred and ninety-eight (798) mother-child dyads living in the Butantã-Jaguaré' region of São Paulo were assessed through a home visit between January and March 2015. Maternal mental health was assessed using the Edinburgh Postnatal Depression Scale (EPDS). Mothers were classified as "possibly depressed" if their EPDS score was between 10 and 13 and as "likely depressed" if their EPDS score was $>13$. The child outcomes analyzed were height, weight, and overall development as assessed by the Ages and Stages Questionnaire (ASQ). Height and weight were age-normalized using WHO growth standards. Stunting was defined as height-for-age $z$-score $(H A Z)<-2$. Obesity was defined as body mass index $z$-score $(B M I Z)>2$. Adjusted and unadjusted linear regression models were used to assess the associations between Edinburgh scores and child outcomes.

Results: No association was found between maternal depression variables and children's height, weight, stunting, and obesity. Positive associations were found between possible depression and ASQ (delta $=0.33$; 95CI 0.11-0.54; $\mathrm{p}$ value $<0.01$ ); no associations were found between likely depression and any of the outcomes analyzed.

Conclusion: The results from this study suggest that symptoms of maternal depression are not associated with delays in child development in the study setting analyzed. Further research will be needed to understand this lack of association: while it is possible that caregivers' mental health did not affect caregiving behavior, it is possible that the effect of maternal depression can vary according to timing, persistence, and intensity. It is also possible that the EPDS instrument may fail to identify mothers with clinical depression, or that children with depressed mothers get increased support from other family members or public early childhood focused programs.

Keywords: maternal depression, linear growth, child development, child obesity. tion, ${ }^{3}$ particularly among women of childbearing age. ${ }^{4}$ Depression rates appear particularly high in low and middleincome countries, particularly among women in extreme poverty; ${ }^{5}$ depression rates in these settings are generally 2 to 3 times higher than those observed in industrial countries. ${ }^{6}$ Among low income Brazilian women the prevalence of depression in the third trimester of pregnancy is $38 \%$; 
during the first 6 months after delivery, $43 \%$ of the women experience at least one episode of depression. ${ }^{7}$

Distress in pregnancy and during the child's first year does not only affect mothers, but is also likely to significantly impact fetal and child development. ${ }^{1}$ Existing evidence suggests that mothers with depressive symptoms are less sensitive and responsive in their daily interactions with their children, are more negative, and use physical punishment. ${ }^{8}$ Both theoretical and empirical work suggest that maternal depression may be particular harmful in early life because children heavily depend on their primary caregivers for care in infancy, and because support and social interactions are particularly important due to this period's high degree of developmental plasticity. ${ }^{3}$ In resource-poor settings, especially in the context of low levels of social support, parenting difficulties are common, further increasing the risk of negative child outcomes. ${ }^{5}$

Observational studies have documented many parenting difficulties associated with maternal depression, including increased hostility, fewer and more negative interactions, less responsiveness or less efficiently communication. ${ }^{9}$ Postpartum depression similarly interferes with many caregiver activities, including breastfeeding, sleep routines, immunization, and safety practices, ${ }^{10,11}$ severely disrupting mother-child relationship. ${ }^{12}$

A growing literature has investigated the association between maternal psychological distress and infant outcomes. ${ }^{1,6}$ Poor maternal mental health has been linked to preterm and low birth weight infant, as well as to poor growth outcomes during the first year of life. ${ }^{4}$ In São Paulo, psychiatric disorders among mothers living in favelas were associated with child underweight. ${ }^{13}$ Some studies have also linked maternal depression and child short stature. Maternal depressive symptoms have also been associated with a higher likelihood of overweight in a population of low-income Brazilian children between 6-24 months of age. ${ }^{14}$ Conceptually, it seems plausible that disruptions in the feeding behavior of depressed mothers could affect both sides of the growth distribution, leading either to stunting or obesity. ${ }^{15}$

A systematic review conducted in 2012 demonstrated that 4 of 5 developmental domains in infants from birth to 1 year of age were adversely influenced by maternal psychological distress (behavior, psychomotor, cognitive and social emotional delay). ${ }^{1}$ Another longitudinal study comprising children of depressed mothers found a raised rate of insecure infant-attachment, impaired cognitive development, specifically in boys, and elevated rate of behavioral and emotional problems. ${ }^{16}$
Many studies, on the other hand, had found no evidences of associations between maternal depression and child development adverse outcomes, such as Santos et al., ${ }^{6}$ Black et al. ${ }^{17}$ Ertel et al. ${ }^{18}$ and Surkan et al. ${ }^{19}$ Grote et al. concluded that maternal postnatal depression may not carry risk at least not in European population. ${ }^{15}$

The controversial findings presented can be a result of diversity in terms of samples, methods and measures. ${ }^{9}$ However, due to the large proportion of depression rates among the population, and its possible consequences to child development, such theme demands further exploration.

The present study aims to understand the associations between maternal depression and child development among a low-resource urban population in Brazil.

\section{Method}

\section{Study setting}

The study was conducted in the Butantã-Jaguaré (BJ) region, which is located in the Western Region of São Paulo. The BJ region has an estimated population of 380,000 . Infant mortality rates in the region vary between 4.4 deaths per 1,000 live births in Morumbi District and 10.3 deaths per 1,000 live births in Vila Sônia District. ${ }^{20,21}$

\section{Study population}

The study population comprised 798 mother-child dyads from the $\mathrm{BJ}$ region, assessed as part of the baseline survey conducted for an ongoing intervention trial (ClinicalTrials.gov NCT02704000). All children in the study were born at the University Hospital (HU-USP), the main public general hospital of the region. The hospital covers the large majority of all women enrolled in public national health system (SUS), and about $40 \%$ of all births in the region. ${ }^{22}$ Nine hundred children born between September 2013 and March 2014 were randomly selected for the intervention trial among all hospital births by mothers living in the $\mathrm{BJ}$ region.

\section{Data}

Data was collected through home visits by trained interviewers between January and March 2015. A total of 900 interviews were conducted. A hundred of them were excluded from this study because the primary caregiver of the child assessed was the child's grandmother; two records were excluded because the Edinburgh Scale was not administered, leaving a final sample of 798 observations.

\section{Exposure variables}

The main exposure variable of interest was maternal depression. Mother's mental health status was assessed us- 
ing the 10-item Edinburgh Postnatal Depression Scale (EPDS). ${ }^{23}$ The scale ranges between 0 and 30; following standard scoring guidelines, ${ }^{23}$ we classified mothers as "possibly depressed" if their score was between 10 and 13 , and as "likely depressed" if their score was greater than 13 .

\section{Outcome variables}

The primary outcome of interest was children's development at 1 year of age. For physical development, age- and gender-normalized height-for-age (HAZ), weight-for-age (WAZ) and body mass index (BMIZ) z-scores were computed using the WHO's anthro software. ${ }^{24}$ Stunting was defined as HAZ < -2; child obesity was defined as BMIZ $>2$. Children's age was adjusted for prematurity. Gestational-length adjusted age was computed as the number of months between the time of the assessment and the child's due date. Children's overall development was assessed using the Brazilian version of the Ages and Stages Questionnaire (BR-ASQ). ${ }^{25}$

\section{Statistical analysis and empirical strategy}

To assess the associations between maternal depression and child development we estimate multivariate linear regression models. The basic model estimated can be described as follows:

$$
y_{i}=\alpha+\beta \text { possible } D_{i}+\text { dlikely }_{i}+X_{i} \gamma+\varepsilon_{i}
$$

where $y_{i}$ is the child outcome of interest, possible $D$ is an indicator for possible depression (EPDS score 10-13) and likely $D$ is an indicator for likely depression (EPDS score $>13)$. $X$ is a vector of maternal and child characteristics. As a first step, we estimate unadjusted as well as adjusted models in the pooled sample; we then estimate these models separately for male and female children, and show non-parametric estimates of the relationship between EPDS scores and child outcomes.

All statistical analysis was performed using the Stata 14 statistical software package. ${ }^{26}$ Ethical clearance for the intervention study was obtained from the University of São Paulo’s Institutional Review Board.

\section{Results}

Figure 1 shows the empirical distribution of Edinburgh Postnatal Depression Scale (EPDS) scores. A total of 100 mothers $(12.5 \%)$ were categorized as possibly depressed (EPDS score 10-13), and 70 mothers (8.8\%) were classified as likely depressed (EPDS score > 13).

Table 1 summarized sample characteristics by EPDS category. Average height-for-age z-score was -0.12 , with

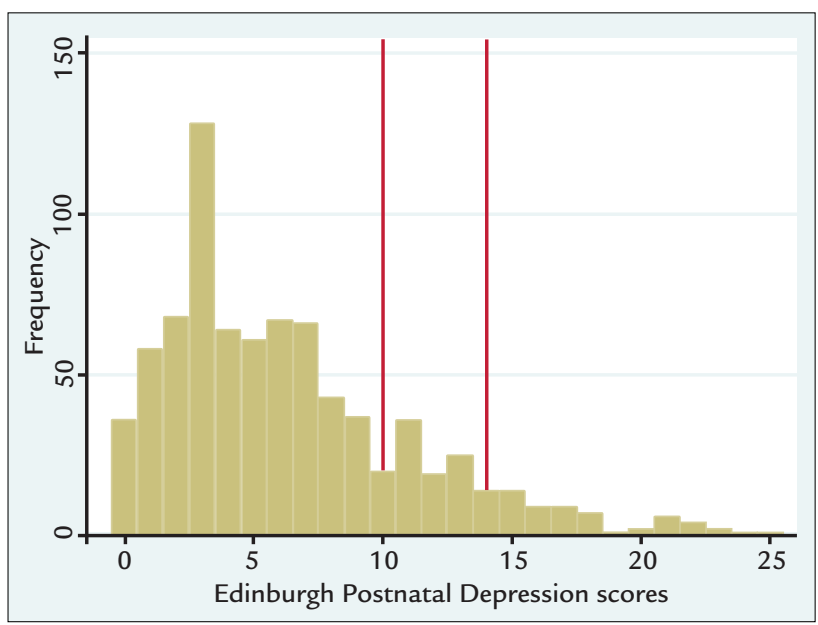

FIGURE 1 Distribution of Edinburgh Postnatal Depression Scale scores.

$7.3 \%$ stunted. Average weight-for-age z-score was 0.44 , with $18.0 \%$ of children falling in the obese category (BMI $z$-score $>2$ ). Average age of children was 12.5 months at the time of the interview; $55 \%$ of children were female, and $1.3 \%$ referred to multiple births. Eight percent $(8.3 \%)$ of children were born prematurely and $13 \%$ had teenage mothers. Most caregivers had secondary education; $16 \%$ had higher education; only $48.6 \%$ of caregivers were married at the time of the interview.

Table 2 shows the results of the multivariable regression analysis. No associations were found between the two depression variables and physical growth variables, including stunting and obesity. For child development (Ages and Stages z-scores), slightly higher scores were found for mothers with possible (but not likely) depression. On average, children of mothers in this group scored 0.33 standard deviations higher than children of mothers with EPDS scores $<10$ (delta $=0.33$, 95CI 0.11-0.54; $\mathrm{p}$-value $<0.01$ ). No associations were found between likely depression and developmental outcomes.

Figure 2 shows polynomial smoothed relationships between our main outcome variables and EPDS scores separately for male and female children. Virtually all trend lines are nearly perfectly flat up to a score of about 15 . Among mothers with an EPDS score of > 15, negative trends were observed for all outcomes (including ASQ) for females; for males, trends seem opposite, with improvements in ASQ, but also improvements in the prevalence of under- and over nutrition. Due to the small sample size in this bracket - only 44 women $(5.5 \%)$ have a score > 15 - none of the observed subgroup differences were statistically significant. 
TABLE 1 Sample characteristics by EPDS category.

\begin{tabular}{|c|c|c|c|c|c|c|c|c|}
\hline \multicolumn{3}{|c|}{$\begin{array}{l}\text { No depression } \\
(\text { EPDS }<10)\end{array}$} & \multicolumn{3}{|c|}{$\begin{array}{l}\text { Possible depression } \\
\text { (EPDS 10-13) }\end{array}$} & \multicolumn{3}{|c|}{$\begin{array}{l}\text { Likely depression } \\
(\text { EPDS > 13) }\end{array}$} \\
\hline Mean & $N$ & SD & Mean & $\mathbf{N}$ & SD & Mean & $\mathbf{N}$ & SD \\
\hline-0.17 & & 1.36 & 0.12 & 11 & 1.52 & 0.05 & 3 & 1.40 \\
\hline 0.08 & 48 & & 0.04 & 4 & & 0.09 & 6 & \\
\hline 0.44 & & 1.25 & 0.42 & & & 0.51 & & 1.08 \\
\hline 0.19 & 106 & & 0.17 & 14 & 0.37 & 0.19 & 13 & \\
\hline-0.05 & & 1.02 & 0.25 & & 0.90 & 0.02 & & 0.86 \\
\hline 12.45 & & 1.87 & 12.57 & & 1.71 & 12.45 & & 1.91 \\
\hline 0.53 & 336 & & 0.65 & 65 & 0.48 & 0.59 & 41 & 0.50 \\
\hline 0.01 & 8 & & 0.00 & 0 & 0.00 & 0.03 & 2 & 0.17 \\
\hline 0.08 & 53 & & 0.03 & 3 & 0.17 & 0.09 & 6 & 0.28 \\
\hline 0.08 & 53 & & 0.09 & 9 & 0.29 & 0.06 & 4 & 0.23 \\
\hline 0.13 & 79 & & 0.17 & 17 & 0.38 & 0.11 & 8 & 0.32 \\
\hline 0.75 & 471 & & 0.69 & 69 & 0.46 & 0.73 & 51 & 0.45 \\
\hline 0.13 & 80 & & 0.14 & 14 & 0.35 & 0.16 & 11 & 0.37 \\
\hline 0.83 & 523 & & 0.85 & 85 & 0.36 & 0.90 & 63 & 0.30 \\
\hline 0.29 & 182 & & 0.33 & 33 & 0.47 & 0.49 & 34 & 0.50 \\
\hline 0.45 & 282 & & 0.41 & 41 & 0.49 & 0.37 & 26 & 0.49 \\
\hline 0.17 & 108 & & 0.18 & 18 & 0.39 & 0.10 & 7 & 0.30 \\
\hline 0.50 & 318 & & 0.43 & 43 & 0.50 & 0.40 & 28 & 0.49 \\
\hline 0.18 & 114 & & 0.29 & 29 & 0.46 & 0.30 & 21 & 0.46 \\
\hline 0.27 & 167 & & 0.26 & 26 & 0.44 & 0.21 & 15 & 0.41 \\
\hline 0.14 & 88 & & 0.09 & 9 & 0.29 & 0.19 & 13 & 0.39 \\
\hline 0.20 & 125 & & 0.24 & 24 & 0.43 & 0.20 & 14 & 0.40 \\
\hline 0.22 & 136 & & 0.12 & 12 & 0.33 & 0.10 & 7 & 0.30 \\
\hline
\end{tabular}

\section{TABLE 2 Adjusted and unadjusted associations between depression scores and child developmental outcomes.}

\begin{tabular}{|c|c|c|c|c|c|}
\hline \multirow[t]{2}{*}{ Outcome } & Height-for-age z-score & Child is stunted & Weight-for-age z-score & Child is obese & Ages and stages z-score \\
\hline & \multicolumn{5}{|l|}{ Unadjusted associations } \\
\hline \multirow{2}{*}{$\begin{array}{l}\text { Possible } \\
\text { depression }\end{array}$} & 0.266 & -0.028 & -0.002 & -0.019 & $0.309 * * *$ \\
\hline & $(0.163)$ & $(0.022)$ & $(0.176)$ & $(0.044)$ & $(0.110)$ \\
\hline \multirow[t]{2}{*}{ Likely depression } & 0.191 & 0.020 & 0.083 & 0.011 & 0.086 \\
\hline & $(0.176)$ & $(0.036)$ & $(0.144)$ & $(0.052)$ & $(0.128)$ \\
\hline Observations & 795 & 795 & 707 & 703 & 602 \\
\hline \multirow[t]{2}{*}{ R-squared } & 0.007 & 0.007 & 0.000 & 0.002 & 0.011 \\
\hline & \multicolumn{5}{|l|}{ Adjusted associations } \\
\hline \multirow{2}{*}{$\begin{array}{l}\text { Possible } \\
\text { depression }\end{array}$} & 0.251 & -0.031 & -0.080 & -0.035 & $0.329 * * *$ \\
\hline & $(0.166)$ & $(0.023)$ & $(0.177)$ & $(0.046)$ & $(0.109)$ \\
\hline \multirow[t]{2}{*}{ Likely depression } & 0.212 & 0.017 & 0.098 & 0.012 & 0.142 \\
\hline & $(0.166)$ & $(0.034)$ & $(0.143)$ & $(0.053)$ & $(0.128)$ \\
\hline Observations & 795 & 795 & 707 & 703 & 602 \\
\hline R-squared & 0.070 & 0.065 & 0.065 & 0.027 & 0.098 \\
\hline
\end{tabular}




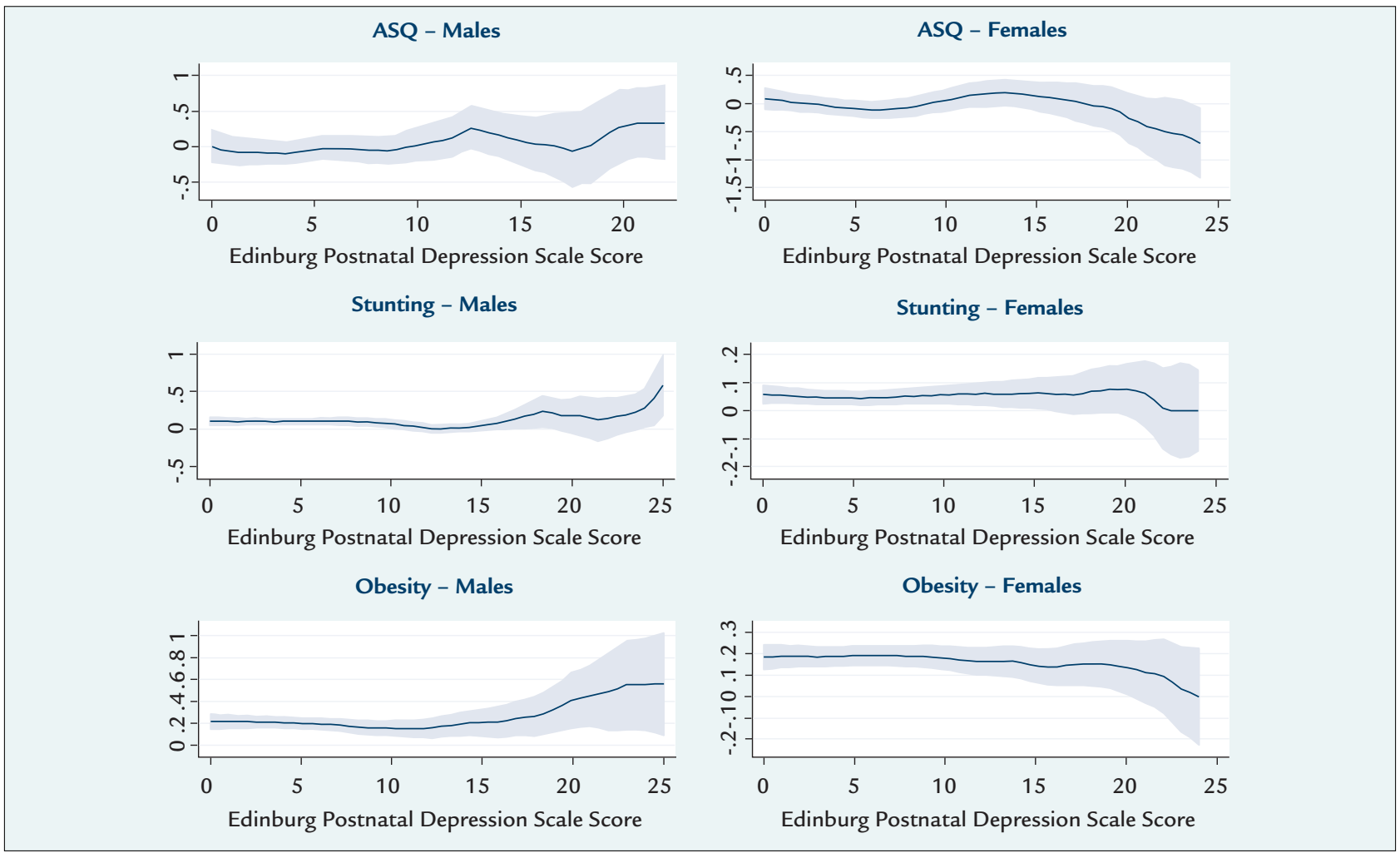

FIGURE 2 Non-parametric trend estimates: Edinburgh Postnatal Depression Scale (EPDS) score, Ages and Stages Questionnaire (ASQ),

stunting and obesity.

\section{Discussion}

The results presented in this paper suggest that symptoms of maternal depression (as measured by the Edinburgh Postnatal Depression Scale) are not associated with child development in the Western Region of São Paulo. While we do not find any associations at all for measures of physical growth and child weight, intermediate EPDS scores actually seem to be positively associated with children's overall development, while only very high EPDS scores appear to be associated with reductions in early childhood development scores, and only for girls.

The general lack of association between maternal depression scores and developmental outcomes is somewhat surprising; while there are studies with similar findings, the majority of the existing literature discussed in the introduction suggests negative associations between maternal depression and children's physical as well as overall development. Several factors potentially contribute to the rather weak overall association observed: first, children were on average only 12 months old when assessed, which makes it relatively difficult to assess cognitive and socio-emotional development of children. In terms of physical growth, most children in the area are doing rather well as evidenced by a stunting rate of only $7 \%$ - large differences in this domain are thus relatively unlikely to occur. Conceptually, maternal mental health may also become more relevant once children become a little bit older and depend more on child-caregiver interactions for stimulation and early learning, and as demonstrated in other studies, the effects of maternal depression during early life can become evident later in life. Second, and related, access to health services is almost universal in the study area, so that children with early developmental problems are likely to have received external support, reducing the differentials between depressed and non-depressed mothers. Last, it is also possible that the relative short EPDS scale is not sensitive enough to clearly distinguish between clinically depressed and healthy mothers; the resulting measurement error will mechanically bias all of our coefficients towards zero. In addition to it, maternal depression was assessed only once, so our analysis cannot distinguish mothers with a persisting depression from the ones with acute episodes, or antenatal depression.

The presented study has several limitations: first, as already mentioned, it relies on the EPDS as primary measure of maternal depression; while the EPDS has been widely used as a screening tool, it is not a direct measure of clinical depression, and thus does not allow us to directly 
compare healthy to clinically depressed mothers. Second, and along similar lines, the study did not conduct a direct assessment of children's cognitive and socio-emotional development. While the ASQ tool used has been validated in Brazil, it relies on maternal self-reports, which may be biased differentially across EPDS groupings. The same obviously does not apply to the anthropometric measures which were taken by study staff as part of the interviews. Last, as it is always the case for cross-sectional studies, we cannot rule out confounding bias. While we use a large set of covariates in our adjusted models, it is possible that omitted variables like maternal family background or maternal social networks affect both maternal health and child outcomes. In most cases, we would expect such omitted factors to improve maternal mental health as well as child well-being; if this was the case, the true associations between maternal mental health and child outcomes would be even more positive than the one reported here.

Despite these limitations, the study has several important strengths: it is, to our knowledge, one of the very few studies assessing the relationship between maternal mental health and child development in a representative urban sample; the large amount of data collected as part of the larger study allows us to control for most environmental factors; the large number of child developmental measures collected allows us to conduct a multi-domain assessment rather than just focusing on nutritional or developmental outcomes.

\section{Conclusion}

Maternal depression is highly common among mothers in poor urban settings. According to our estimates, depression seems possible for $12.5 \%$ of mothers and likely for $8.8 \%$ of mothers. The results of this study suggest that depression symptoms are not generally associated with reductions in child development at age one. Negative associations seem plausible for mothers with higher EPDS scores; our study was not powered to detect such differences. Overall, the effect of maternal depression on child development appears to vary substantially depending on local context, and, as suggested by Claessens et al., ${ }^{3}$ the timing, persistence and intensity of depressive symptoms.

\section{References}

1. Kingston D, Tough S, Whitfield H. Prenatal and postpartum maternal psychological distress and infant development: a systematic review. Child Psychiatry Hum Dev. 2012; 43(5):683-714.
2. Prince M, Patel V, Saxena S, Maj M, Maselko J, Phillips MR, et al. No health without mental health. Lancet. 2007; 370(9590):859-77.

3. Claessens A, Engel M, Curran FC. The effects of maternal depression on child outcomes during the first years of formal schooling. Early Child Res Q. 2015; 32(3rd Quarter):80-93

4. Atif N, Lovell K, Rahman A. Maternal mental health: The missing " $m$ " in the global maternal and child health agenda. Semin Perinatol. 2015; 39(5):345-52.

5. Parsons CE, Young KS, Rochat TJ, Kringelbach ML, Stein A. Postnatal depression and its effects on child development: a review of evidence from low- and middle-income countries. Br Med Bull. 2012; 101(1):57-79.

6. Santos IS, Matijasevich A, Domingues MR, Barros AJ, Barros FC. Longlasting maternal depression and child growth at 4 years of age: a cohort study. J Pediatr. 2010; 157(3):401-6.

7. Da-Silva VA, Moraes-Santos AR, Carvalho MS, Martins ML, Teixeira NA. Prenatal and postnatal depression among low income Brazilian women. Braz J Med Biol Res. 1998; 31(6):799-804.

8. McLearn K, Minkovitz C, Strobino DM, Marks E, Hou W. The timing of maternal depression symptoms and mother's parenting practices with young children: implications for pediatric practice. Pediatrics. 2006; 118(1):e174-82.

9. Lovejoy MC, Graczyk PA, O’Hare E, Neuman G. Maternal depression and parenting behavior: a meta-analytic review. Clin Psychol Rev. 2000; 20(5):561-92.

10. Lefkovics E, Baji I, Rigó J. Impact of maternal depression on pregnancies and on early attachment. Infant Ment Health J. 2014; 35(4):354-65.

11. Stewart RC. Maternal depression and infant growth: a review of recent evidence. Matern Child Nutr. 2007; 3(2):94-107.

12. Apter-Levi Y, Pratt M, Vakart A, Feldman M, Zagoory-Sharon O, Feldman R. Maternal depression across the first years of life compromises child psychosocial adjustment; relations to child HPA-axis functioning. Psychoneuroendocrinology. 2016; 64:47-56.

13. de Miranda CT, Turecki G, Mari Jde J, Andreoli SB, Marcolim MA, Goihman $\mathrm{S}$, et al. Mental health of the mothers of malnourished children. Int J Epidemiol. 1996; 25(1):128-33.

14. Surkan PJ, Kawachi I, Ryan LM, Berkman LF, Carvalho Vieira LM, Peterson KE. Maternal depressive symptoms, parenting self-efficacy, and child growth. Am J Public Health. 2008; 98(1):125-32.

15. Grote V, Vik T, von Kries R, Luque V, Socha J, Verduci E, et al.; European Childhood Obesity Trial Study Group. Maternal postnatal depression and child growth: a European cohort study. BMC Pediatr. 2010; 10:14.

16. Tomlinson M, Cooper PJ, Stein A, Swartz L, Molteno C. Post-partum depression and infant growth in a South African peri-urban settlement Child Care Health Dev. 2006; 32(1):81-6.

17. Black MM, Baqui AH, Zaman K, El Arifeen S, Black RE. Maternal depressive symptoms and infant growth in rural Bangladesh. Am J Clin Nutr. 2009; 89(3):951S-7S

18. Ertel KA, Koenen KC, Rich-Edwards JW, Gillman MW. Maternal depressive symptoms not associated with reduced height in young children in a US prospective cohort study. PLoS One. 2010; 5(10):e13656.

19. Surkan PJ, Ryan LM, Carvalho Vieira LM, Berkman LF, Peterson KE. Maternal social and pyschological conditions and physical growth in low-income children in Piauí, Northeast Brazil. Soc Sci Med. 2007; 64(2):375-88.

20. Sistema de Informação Sobre Mortalidade (SIM) [Internet]. 2014 [cited 2014 April]. Available from: http://svs.aids.gov.br/cgiae/sim/.

21. Prefeitura do Município de São Paulo. Mortalidade Infantil no Municipio de São Paulo 2014.

22. Prefeitura de SP Saúde. Informações em saúde - Mãe Paulistana. 2012

23. Cox JL, Holden JM, Sagovsky R. Detection of postnatal depression. Development of the 10-item Edinburgh Postnatal Depression Scale. Br J Psychiatry. 1987; 150:782-6.

24. WHO. Anthro Software for assessing growth and development of the world's children. Geneva: WHO; 2006.

25. Filgueiras A, Pires P, Maissonette S, Landeira-Fernandez J. Psychometric properties of the Brazilian-adapted version of the Ages and Stages Questionnaire in public child daycare centers. Early Hum Dev. 2013; 89(8):561-76.

26. StataCorp. Stata Statistical Software: Release 14. College Station: StataCorp LP; 2015. 